\title{
On a Non-Ćlassical Boundary Value Problem in Linear Plané Elasticity
}

\author{
J. MaUL ?
}

Dedicated to my teacher Professor Dr. H. Beckert on the occassion of his 65. birthday

In dér Arbeit wird ein nicht-klassisches Randwertproblem der ebenen linearen Elastizitätstheorie betrachtet. Durch Zurückführung auf singuläre Integralgleichungen werden die Fredholmschen Sätze bewiesen. Mit funktionentheoretischen Methoden werden der Einheitskreis sowie Epitrochoiden eingehend untersucht. Es wird bewiesen, daß zu jeder natürlichen Zahl $n$ und innerhalb jeder beliebig kleinen Umgebung des Einheitskreises eine solche Epitrochoide existiert, daß das homogene nicht-klassische Randwertproblem für dieses Gébiet mindestens $n$ linear unabhängige Lösungen besitzt.

В работе рассматривается неклассическая задача плоской линей теории упругости. Сведением к сингулярным интегральным ураввениям доказываются теоремы фредгольма. Методом теории функций одно комплексно переменно исследуются случаи единичного круга и әпитрохоидов. Доказывается, что для проиввольного натурального числа $n$ и внутри произвольной окрестности единичного круга суцествует тако рохоид, что однородная неклассическая краевая задача Дирихле в̈ этой области имеет по меньшей мере $n$ линейо-независимых решений.

The paper deals with a non-classical boundary value problem of linear plane elasticity. With the aid of singular integral equations, Fredholm's theorems are proved. Using complex variable techniques, the problem is considered for the unit circle and epitrochoids. It is proved that for every positive. integér $n$ and within every arbitrarily small vicinity of the unit circle there exists such an epitrochoid for which the homogeneous non-classical problem allows at least $n$. linearly independent solutions.

In the present paper, a non-classical boundary value problem of linear plane elastostatics is treated. In $\S 1$ this problem is formulated with remarks on its mechanical significance: 'The proof of Fredholm's theorems is sketched in $\S 2$. Using complex variable methods, we consider the related homogeneous problem in $\S \S 3-7$. In this way we can realize a remárkable sensibility of the considered non-classical problem in respect to the variation of the domain. Indeed, we shall prove that in every small vicinity of the unit circle there are such domains for which the homogeneous problem allows an arbitrarily large number of linearly independent solutions. This property is quite interesting with respect to the simultaneous validity of Fredholm's theorems. Notice that a similar behaviour is unknown in the theory of related plane-problems for a single elliptic differential equation of second order, for instance, the oblique derivative problem. In this case the number of linearly independent solutions of the corresponding homógeneous problems depends only on the index of the problem; but not on the domain (Horrich [3], Frchera [2], Schubert [11]). Another non: classical problem of plane elasticity has been studied in $[7,8]$ by the author. 


\section{$\$ 1$ Statement of the non-classical problem}

Let $D \subset \mathbf{R}^{2}$ be a given bounded simply connected domain with boundary $L=\partial D$ $\epsilon C^{1, \beta}(0<\beta \leqq 1)$. Let $D$ be occupied by an elastic isotropic and homogeneous body in the sense of plane elasticity with the Lamé constants $\lambda, \dot{\mu}>0$. We consider the problem to solve the system

$$
\left.\frac{\partial}{\partial x_{j}} \sigma_{i j}(\mathbf{x})=0 \quad(\mathrm{x} \in D) ; i, j=1,2 \text {, summing up } j !\right) \quad, \quad !
$$

of linear plane elastostatics without volume forces with the boundary. conditions

$$
\begin{aligned}
& -\sigma_{1}{ }^{(\mathbf{n})}(\mathrm{z}) \cos \left(\mathbf{n}(\mathbf{z}), \mathbf{x}_{2}\right)+\sigma_{2}{ }^{(\mathbf{n})}(\mathbf{z}) \cos \left(\mathbf{n}(\mathbf{z}), \mathbf{x}_{1}\right)=0 \\
& -u_{1}(\mathbf{z}) \cos \left(\mathbf{n}(\mathbf{z}), \mathbf{x}_{2}\right)+u_{2}(\mathbf{z}) \cos \left(\mathbf{n}(\mathbf{z}), \mathbf{x}_{1}\right)=g(\mathbf{z}) \quad(\mathbf{z} \in L) .
\end{aligned}
$$

In these formulas, $\mathbf{n}(\mathrm{z})$ means the outward normal of $L$ at $\mathrm{z}, g$ is a given function of the class $C^{1, \alpha}(L)(0<\alpha<\beta \leqq 1)$, and $\sigma_{i}^{(\mathrm{n})}$ mean the components of the stress vector $\sigma^{(n)}=\left(\sigma_{1}^{(n)}, \sigma_{2}^{(n)}\right)$ in the Cartesian system, which satisfy the relations

$$
\sigma_{i}^{(\mathbf{n})}=\sigma_{i j} \cos \left(\mathbf{n}, \mathbf{x}_{j}\right) \quad(i, j=1,2 ; \text { summing up } j) .
$$

$u_{i}$ in $(2 \mathbf{b})$ are the components of the displacement vector $\mathbf{u}=\left(u_{1}, u_{2}\right)$. The relations between $u$ and $\sigma_{i}$, are given' by the generalized Hooke's law

$$
\sigma_{i j}=\lambda \cdot \theta \delta_{i j}+\mu\left(\frac{\partial u_{i}}{\partial x_{j}}+\frac{\partial u_{j}}{\partial x_{i}}\right), \quad \theta=\frac{\partial u_{1}}{\partial x_{1}}+\frac{\partial u_{2}}{\partial x_{2}} .
$$

The mechanical meaning of the condition (2) will be apparent from the consideration of the "extreme fibre"' of the body along ' $L$. In virtue of $(2 \mathrm{a})$, the extreme fibre is tracted by pure tension or pressure, but not by shear stresses. The tangential displacement of the extreme fibre is given by $(2 \mathrm{~b})$.

In terms of displacements, the problem (1); (2) can be formulated as follows: To find a solution $\mathbf{u}=\left(u_{1}, u_{2}\right)$ of the equations

$$
\mu \Delta \mathbf{u}+(\hat{\lambda}+\mu) \operatorname{grad} \operatorname{div} \mathbf{u}=\mathbf{0}
$$

with boundary conditions (2), where the connection between the stress vector and the displacements is given by

$$
\begin{aligned}
& \boldsymbol{\sigma}^{(\mathbf{n})}=\left(\dot{\sigma}_{1}^{(\mathbf{n})}, \sigma_{2}{ }^{(\mathbf{n})}\right)=\mathscr{T}(\mathbf{n}) \mathbf{u} \\
& \dot{T}(\mathbf{n}) \mathbf{u}=2 \mu \frac{\partial \mathbf{u}}{\partial \mathbf{n}}+\lambda \mathbf{n} \operatorname{div} \mathbf{u}+\mu\left(\frac{\partial u_{1}}{\partial x_{2}}-\frac{\partial u_{2}}{\partial x_{1}}\right)\left(-n_{2}, n_{1}\right), \\
& \mathbf{n}=\left(n_{1}, n_{2}\right)=\left(\cos \left(n, \mathbf{x}_{1}\right), \cos \left(\mathbf{n}, \mathbf{x}_{2}\right)\right) .
\end{aligned}
$$

$\mathscr{T}(\mathbf{n})$ is called operator of normal stresses.

\section{' 2 Fredholm's theorems}

Fredholm's theorems for the problem (1), (2) (or (5), (2)) can be proved by the aid of a potential approach with the framework of [6], which is sketched below. The considerations are restricted to such solutions $\mathbf{u}$ of problem (5), (2) which belong to the class $C^{1, \alpha}(\bar{D}) \cap C^{2}(D)$. For such a solution $\mathbf{u}$ we can find a vectorial density $\boldsymbol{\Phi}(\mathbf{y})$ 
$=\left(\Phi_{1}(y), \Phi_{2}(y)\right)$ of the class $C^{0 . \alpha}(L)$ which satisfies the formula

$$
\mathbf{u}(\mathbf{x})=\mathbf{V}(\mathbf{x}, \boldsymbol{\Phi})=\frac{1}{\pi} \int_{\boldsymbol{L}} \boldsymbol{\Gamma}(\mathbf{x}-\mathbf{y}) \boldsymbol{\Phi}(\mathbf{y}) d s, \quad \mathbf{x} \in D .
$$

Furthermore, the density $\Phi$ is uniquely determined by the displacements u [6]. The matrix $\Gamma(x-y)$ in.(7) is the Kelvin-Somigliana fundamental solution, given by

$$
\begin{aligned}
& \Gamma(\mathrm{z})=\left[\Gamma_{i j}(\mathrm{z})\right]_{i, j=1.2}, \\
& \Gamma_{i j}(\mathrm{z})=a \ln \frac{k}{|\mathrm{z}|} \delta_{i j}+b \frac{z_{\mathrm{i}} z_{j}}{|\mathrm{z}|^{2}}, \quad k>0, \\
& a=\frac{\lambda+3 \mu}{2 \mu(\lambda+2 \mu)}, \quad b=\frac{\lambda+\dot{\mu}}{2 \mu(\lambda+2 \mu)} .
\end{aligned}
$$

The real constant $k$ must not coincide with an exceptional value, but for a given domain $D$ there exist no more than 2 exceptional values [6: p. 34 Hilfssatz 9.2]:- The main properties of the simple layer potentia] (7) can be found in [6]; for instance the jump relation

$$
\mathscr{T}(\mathbf{n}) \mathbf{V}(\mathbf{z} ; \boldsymbol{\Phi})^{ \pm}=\lim _{\mathbf{x} \rightarrow D \pm \mathbf{D} \in L} \mathscr{T}(\mathbf{n}) \mathbf{V}(\mathbf{x} ; \boldsymbol{\Phi})= \pm \boldsymbol{\Phi}(\mathbf{z})+\frac{1}{\pi} \int_{l} \boldsymbol{\Gamma}_{I}^{*}(\mathbf{z}, \mathbf{y}) \boldsymbol{\Phi}(\mathbf{y}) d s
$$

with

$$
\begin{aligned}
& D^{+}=D, \quad D^{-}=C \bar{D}, \quad \boldsymbol{\Gamma}_{I}^{*}=\left[\Gamma_{I_{i}}^{*}\right]_{i, j=1,2}, \\
& \left(\Gamma_{I_{14}}^{*}(\mathbf{z}, \mathbf{y}), \Gamma_{I_{3 i}}^{*}(\mathbf{z}, \mathbf{y})\right)=\mathscr{T}\left(\mathbf{n}_{\mathbf{z}}\right)\left(\Gamma_{1 i}(\mathbf{z}-\mathbf{y}), \Gamma_{2 i}(\mathbf{z}-\mathbf{y})\right) \quad(i=1,2)
\end{aligned}
$$

and the formula

$$
\frac{d}{d s_{\mathrm{z}}} \mathbf{V}(\mathbf{z} ; \Phi)=\frac{1}{\pi} \int_{L} \frac{d}{d s_{\mathbf{z}}} \boldsymbol{\Gamma}(\mathbf{z}-\mathbf{y}) \boldsymbol{\Phi}(\mathbf{y}) d s
$$

which are valid under the assumptions $L \in C^{1, \beta}, \Phi \in C^{0 . \alpha}(L)$. Moreover, the displacements $u$ of (7) are solutions of (5) in the classical sense.

In order to satisfy the boundary conditions, $\Phi$ must be a solution of the integral equation' system

$$
\begin{aligned}
& -n_{2}(\mathrm{z}) \Phi_{1}(\mathrm{z})+n_{1}(\mathrm{z}) \Phi_{2}(\mathrm{z}) \\
& +\frac{1}{\pi} \int_{L}\left\{K_{11}(\mathrm{z}, \mathrm{y}) \Phi_{1}(\mathrm{y})+K_{12}(\mathrm{z}, \mathrm{y}) \Phi_{2}(\mathrm{y})\right\} d s_{\mathrm{y}}=0 \\
& \frac{1}{\pi} \int_{\mathrm{L}}\left\{K_{21}(\mathrm{z}, \mathrm{y}) \Phi_{1}(\mathrm{y})+K_{22}(\mathrm{z}, \mathrm{y}) \Phi_{2}(\mathrm{y})\right\} d s_{\mathrm{y}}=g(\mathrm{z})
\end{aligned}
$$

where the kernel functions $K_{i j}$ are given by

$$
\begin{aligned}
& K_{11}(\mathrm{z}, \mathrm{y})=-n_{2}(\mathrm{z}) \Gamma_{I_{11}}^{*}(\mathrm{z}, \mathrm{y})+n_{1}(\mathrm{z}) \Gamma_{I_{12}}^{*}(\mathrm{z}, \mathrm{y}) \\
& K_{12}(\mathrm{z}, \mathrm{y})=-n_{2}(\mathrm{z}) \Gamma_{I_{13}}^{*}(\mathrm{z}, \mathrm{y})+n_{1}(\mathrm{z}) \Gamma_{I_{12}}^{*}(\mathrm{z}, \mathrm{y}) \\
& K_{21}(\mathrm{z}, \mathrm{y})=-n_{2}(\mathrm{z}) \Gamma_{11}(\mathrm{z}-\mathrm{y})+n_{1}(\mathrm{z}) \dot{\Gamma}_{21}(\mathrm{z}-\mathrm{y}) \\
& K_{22}(\mathrm{z}, \mathrm{y})=-\dot{n}_{2}(\mathrm{z}) \Gamma_{12}(\mathrm{z}-\mathrm{y})+n_{1}(\mathrm{z}) \Gamma_{22}(\mathrm{z}-\mathrm{y})
\end{aligned}
$$


The equivalence between (2), (5) and $(9 \mathrm{a}),(9 \mathrm{~b})$ follows from the results of [6]. Furthermore, the equation $(9 \mathrm{~b})$ is subjected to the operator

$$
\Omega=\Omega\left(\frac{d}{d s}\right)=\left(\frac{d}{d s}+1\right), \quad s-\text { arc length parameter. }
$$

Thus we obtain the equation

$$
\begin{aligned}
& \int_{L}\left\{\frac{d}{d s_{\mathbf{z}}} K_{21}(\mathbf{z}, \mathbf{y}) \Phi_{1}(\mathbf{y})+\frac{d}{d s_{\mathbf{z}}} K_{22}(\mathbf{z}, \mathbf{y}) \Phi_{\mathbf{2}}(\mathbf{y})\right\} d s_{\mathbf{z}} \\
& +\int_{L}\left\{K_{2 \mathrm{i}}(\mathbf{z}, \mathbf{y}) \Phi_{1}(\mathbf{y})+K_{22}(\mathbf{z}, \mathbf{y}) \Phi_{2}(\mathbf{y})\right\} d s_{\mathbf{y}}=\frac{d}{d s} g(\mathbf{z})+g(\overline{\mathbf{z}}) .
\end{aligned}
$$

Using the considerations of $[4:$ p. 113], one can easily prove that the equations (9 b) and $\left(9 b^{\prime}\right)$ are equivalent for $\Phi \in C^{0, \alpha}(L)$. 'The system $(9 a),\left(9 b^{\prime}\right)$ is a singular integral equation system. In the complex $\zeta$-plane $\left(\zeta=z_{1}+i z_{2}\right)$, the system $(9 \mathrm{a}),\left(9 \mathrm{~b}^{\prime}\right)$ can be splitted into the terms

$$
\mathbf{A}(\zeta) \boldsymbol{\Phi}(\zeta)+\frac{\mathbf{B}(\zeta)}{\pi i} \int_{\boldsymbol{L}} \frac{\Phi(\tau)}{\tau-\zeta} d \tau+\mathscr{C} \boldsymbol{\Phi}(\zeta)=\mathbf{F}(\zeta)
$$

where $\mathscr{C}$ is a completely continuous operator and

$$
\mathbf{A}(\zeta)=\left[\begin{array}{cc}
-n_{2}(\zeta) & n_{1}(\zeta) \\
0 & 0
\end{array}\right], \quad \dot{\mathbf{B}(\zeta)}=\left[\begin{array}{cc}
c n_{1}(\zeta) & c n_{2}(\zeta) \\
-a n_{2}(\zeta) & a n_{1}(\zeta)
\end{array}\right], \quad C=\frac{\mu}{\lambda+2 \mu}
$$

Because of

$$
\operatorname{det}[\mathbf{A}+\mathbf{B}]=\left|\begin{array}{ll}
-n_{2} \pm c n_{1} & 1 n_{1} \pm c n_{2} \\
\mp a n_{2} & \pm a n_{1}
\end{array}\right|=a c=\text { const. } \neq 0
$$

the system $(9 a),\left(9 b^{\prime}\right)$ is of regular type in the sense of MuskrelishviLI [10] with index $x=0$.

Consequently, Fredholm's theorems are true for $(9 \mathrm{a}),\left(9 \mathrm{~b}^{\prime}\right)$ and, in virtue of the equivalence between! $(2),(5)$ and $(9 \mathrm{a}),(9 \mathrm{~b})$, we get the same result for the boundary value problem (5), (2) (or (1), (2); respectively). Particularly, there exist at most a finite number of solutions of the homogeneous problem (1),.(2) (with $g=0$ ).

In the following, we will determine explicitely the number $h=h(D)$ of linearly independent solutions of the homogeneous problem (1), (2) for some special domains $D$ by using complex variable methods and conformal mapping technique.

\section{$\$ 3$ Fundamental formulas}

In order to apply complex variable methods, we make use of some widely used formulas. Let $\omega(z)$ be the conformal mapping from the unit circle in the complex $z$-plane to the domain $D$ in the $\zeta$-plane. Then we can introduce curvilinear rectangular co-ordinates $\dot{\varrho} \vartheta$ in $D$, which arise by mapping of the polar co-ordinates $\dot{\varrho}, \dot{\vartheta}$ of the unit circle. Furthermore, it is well-known' in plane elasticity that there exist two holomorphic functions $\ddot{\varphi}_{1}(\zeta), \psi_{1}(\zeta)$ which describe the deformation completely. These functions are in one-to-one correspondence to the holomorphic functions 
$\varphi(z)=\varphi_{1}(\omega(z)), \quad \psi(z)=\psi_{1}(\omega(z))$. By setting $\Phi_{1}(\zeta)=\varphi_{1}^{\prime}(\zeta), \Psi_{1}(\zeta)=\dot{\Psi}_{1}^{\prime}(\zeta), \Phi(z)$ $=\Phi_{1}(\omega(z)), \Psi(z)=\Psi_{1}(\omega(z))$, one gets

$$
\varphi^{\prime}(z)=\omega^{\prime}(z) \Phi(z), \quad \psi^{\prime}(z)=\dot{\omega}^{\prime}(z) \Psi(z) .
$$

The following formulas were deduced e.g. in [9]:

$$
\begin{gathered}
\sigma_{\rho \ell}-i \sigma_{\rho \theta}=\Phi(z)+\overline{\Phi(z)}-\frac{z^{2}}{\varrho^{2} \overline{\omega^{\prime}(z)}}\left(\overline{\omega(z)} \Phi^{\prime}(z)+\omega^{\prime}(z) \Psi(z)\right) \\
\vdots \\
4 \mu\left|\omega^{\prime}(z)\right|\left(u_{\rho}+i u_{\theta}\right)=\frac{\bar{z}}{\varrho} \overline{\omega^{\prime}(z)}\left(x \varphi(z)-\frac{\omega(z)}{\overline{\omega^{\prime}(z)}} \overline{\varphi^{\prime}(\dot{z})}-\overline{\psi(z)}\right) .
\end{gathered}
$$

Here $\sigma_{\rho e}, \sigma_{\rho \theta}$ mean the components of the stress tensor with respect to the Cartesian system in the considered point $\zeta \in D$, whose axes have the directions of the $\varrho$ - and $\vartheta$-co-ordinate lines. $u_{e}, u_{\theta}$ are the corresponding components of the displacement vector. Moreover, we can always assume $\varphi(0)=0$. Setting $\varrho=1$, we get the boundary values of the expressions (12). Now, the homogeneous problem (1), (2) $(g=0)$ can be considered as a problem for determination of the analytic functions $\varphi(z), \psi(z)$ in the unit circle from the boundary conditions

$$
\begin{aligned}
& t^{2} \omega^{\prime}(t)\left[\overline{\omega(t)} \Phi^{\prime}(t)+\omega^{\prime}(t) \Psi(t)\right] \\
& -\bar{t}^{2} \overline{\omega^{\prime}(t)}\left[\omega(t) \overline{\Phi^{\prime}(t)}+\overline{\omega^{\prime}(t)} \overline{\Psi(t)}\right]=0, \\
& \bar{i} \overline{\omega^{\prime}(t)}\left[x \varphi(t)-\frac{\omega(t)}{\overline{\omega^{\prime}(t)}} \overline{\varphi^{\prime}(t)}-\overline{\psi(t)}\right] \\
& -t \omega^{\prime}(t)\left[x \overline{\varphi(t)}-\frac{1}{\omega^{\prime}(t)} \varphi^{\prime}(t)-\psi(t)\right]=0 \text { for }|t|=1 .
\end{aligned}
$$

In the next section we consider the boundary value problem (13) for the mapping function $\omega(z) \doteq z+q z^{p}, q \in \mathbf{R}$. The corresponding domains $D$ are epitrochoids. In consequence of the conformity. we have the condition $\omega^{\prime}(z) \neq 0$ for $|z| \leqq 1$. This implies $|q|<1 / p$.

\section{$\$ 4$. The homogeneous problem in the case $\omega(z)=z+q z^{p}$}

A. Now; we consider the problem (13) in the special case $\omega(z)=z+q z^{p}(p \in \mathbf{N}$; $p \geqq 2$ ). Because of $t \bar{t}=1$ on the unit circle, the boundary conditions become

$$
\begin{aligned}
& t^{2} \omega^{\prime}(t)\left[\bar{\omega}\left(\frac{1}{t}\right) \Phi^{\prime}(t)+\omega^{\prime}(t) \Psi(t)\right] \\
& -\frac{1}{t^{2}} \bar{\omega}^{\prime}\left(\frac{-1}{t}\right)\left[\omega(t) \overline{\Phi^{\prime}(t)}+\bar{\omega}^{\prime}\left(\frac{1}{t}\right) \overline{\Psi(t)}\right]=0 \\
& \frac{x}{t} \bar{\omega}^{\prime}\left(\frac{1}{t}\right) \varphi(t)+t \bar{\omega}\left(\frac{1}{t}\right) \varphi^{\prime}(t)+t \omega^{\prime}(t) \bar{\psi}(t) \\
& -x t \omega^{\prime}(t) \varphi(t)-\frac{\omega(t)}{t} \overline{\varphi^{\prime}(t)}-\frac{\bar{\omega}^{\prime}\left(\frac{1}{t}\right)}{t} \overline{\psi(t)}=0 \text { for }|t|=1
\end{aligned}
$$

1) In the case of plane deformation we have $x=\frac{\lambda+3 \mu}{\lambda+\mu}=3-4 \sigma$, in the case of generalized plane stress $x=\frac{5 \lambda+6 \mu}{3 \lambda+2 \mu}=\frac{3-\sigma}{1+\sigma}\left(\sigma-\right.$ Poisson's ratio, $\left.\frac{1}{4} \leq \sigma<\frac{1}{2}\right)$. 
Using the construction of Schwarz' reflection principle, the holomorphic functions $\varphi(z), \psi(z), \Phi^{\prime}(z), \Psi(z)$, defined on the unit circle, relate to the holomorphic functions $\bar{\varphi}\left(\frac{1}{z}\right), \bar{\psi}\left(\frac{1}{z}\right), \bar{\Phi}^{\prime}\left(\frac{1}{z}\right), \bar{\Psi}\left(\frac{1}{z}\right)$ in the exterior of the unit circle. The boundary values of these functions are given by $\bar{\varphi}\left(\frac{1}{t}\right)=\overline{\varphi(t)}, \ldots, \bar{\Psi}\left(\frac{1}{t}\right)=\overline{\Psi(t)}$. With the piece-
wise analytic functions

$$
\Omega_{1}(z)=\left\{\begin{array}{l}
z^{2} \omega^{\prime}(z)\left[\bar{\omega}\left(\frac{1}{z}\right) \Phi^{\prime}(z)+\omega^{\prime}(z) \Psi(z)\right] \text { for }|z|<1 \\
\frac{\bar{\omega}^{\prime}\left(\frac{1}{z}\right)}{z^{2}}\left[\omega(z) \bar{\Phi}^{\prime}\left(\frac{1}{z}\right)+\bar{\omega}^{\prime}\left(\frac{1}{z}\right) \bar{\Psi}\left(\frac{1}{z}\right)\right] \text { for }|z|>1
\end{array}\right.
$$

and

$$
\Omega_{2}(z)=\left\{\begin{array}{l}
\frac{x}{z} \bar{\omega}^{\prime}\left(\frac{1}{z}\right) \varphi(z)+z \bar{\omega}\left(\frac{1}{z}\right) \varphi^{\prime}(z)+z \omega^{\prime}(z) \psi(z) \text { for }|z|<1^{\prime} \\
x z \omega^{\prime}(z) \bar{\varphi}\left(\frac{1}{z}\right)+\frac{1}{z} \omega(z) \bar{\varphi}^{\prime}\left(\frac{1}{z}\right)+\frac{1}{z} \bar{\omega}^{\prime}\left(\frac{1}{z}\right) \bar{\psi}\left(\frac{1}{z}\right) \text { for }|z|>1,
\end{array}\right.
$$

the boundary conditions (14) are transformed to

$$
\Omega_{1}{ }^{+}(t)-\Omega_{1}{ }^{-}(t)=0, \quad \Omega_{2}{ }^{+}(t)-\Omega_{2}{ }^{-}(t)=0 .
$$

According to (15a) and (15b), the analytic functions $\Omega_{1}(z)$ and $\Omega_{2}(z)$ have poles of the order $(p-2)$ and $p-1)$, respectively, at the origin and at infinity. Thus, the general solution of the jump problems (16) is given by

$$
\begin{aligned}
& \Omega_{i}(z)=R_{i}(z) \quad(i=1,2), \\
& R_{1}(z)=\frac{b_{p-2}}{z^{p-2}}+\frac{b_{p-3}}{z^{p-3}}+\cdots+\frac{b_{1}}{z}+b_{0}+\tilde{b}_{1} z+\cdots+\tilde{b}_{p-3} z^{p-3}+\tilde{b}_{p-2} z^{p-2}, \\
& R_{2}(z)=\frac{c_{p-1}}{z^{p-1}}+\frac{c_{p-2}}{z^{p-2}}+\cdots+\frac{c_{1}}{z}+c_{0}+\tilde{c}_{1} z+\cdots+\tilde{c}_{p-2} z^{p-2}+\tilde{c}_{p-1} z^{p-1}
\end{aligned}
$$

with arbitrary complex constants $b_{i}, \tilde{b}_{j}, c_{k}, \tilde{c}_{l}$. Bearing in mind (15a) and (15 b), one $\begin{aligned} & \text { obtains } \\ & \text { implies }\end{aligned} \bar{\Omega}_{i}\left(\frac{1}{z}\right)=\Omega_{i}(z) \quad(i=1,2)$. Consequently, we have $\bar{R}_{i}\left(\frac{1}{z}\right)=R_{i}(z)$, which

$$
\begin{aligned}
& \tilde{b}_{i}=\bar{b}_{i} \quad(i=1, \ldots, p-2), \quad \tilde{c}_{j}=\bar{c}_{j} \quad(j=1, \ldots, p-1), \\
& b_{0}, c_{0}-\text { real constants. }
\end{aligned}
$$

B. Further restrictions for the constants $c_{i}, b_{k}$ can be deduced if we pay attention to the connection between (15 a) and (15 b). In order to establish these restrictions, we consider the following Taylor expansions in the neighbourhood of the origin:

$$
\begin{aligned}
\varphi(z)= & K_{1} z+K_{2} z^{2}+\cdots+K_{p} z^{p}+O\left(z^{p+1}\right) \quad(\varphi(0)=0) \\
\Phi(z)= & \frac{\varphi^{\prime}(z)}{\omega^{\prime}(z)}=\left(K_{1}+2 K_{2} z+\cdots+(p+1) K_{p+1} z^{p}+\cdots\right) \\
& \times\left(1-p q z^{p-1}+(p q)^{2} z^{2(p-1)}-\cdots\right) \\
= & K_{1}+2 K_{2} z+\cdots+(p-1) K_{p-1} z^{p-2}+p\left(K_{p}-q K_{1}\right) z^{p-1} \\
& +\left[(p+1) K_{p+1}-2 p q K_{2}\right] z^{p}+O\left(z^{p+1}\right)
\end{aligned}
$$




$$
\begin{aligned}
\Phi^{\prime}(z)= & 2 K_{2}+6 K_{3} z+\cdots+(p-2)(p-1) K_{p-1} z^{p-3} \\
& +(p-1) p\left(K_{p}-q K_{1}\right) z^{p-2} \\
& +p\left[(p+1) K_{p+1}-2 p q K_{2}\right] z^{p-1}+O\left(z^{p}\right) \quad \text { for } \quad p \geqq 3
\end{aligned}
$$

but.

$$
\Phi^{\prime}(z)=\left(2 K_{2}-2 q K_{1}\right)+2\left(3 K_{3}-4 q K_{2}+4 q^{2} K_{1}\right) \cdot z+O\left(z^{2}\right) \text { for } p=2 \text {. }
$$

Comparing the first coefficients of the Laurent expansions at the origin on both sides of (17), one gets the linear equations

$$
\begin{aligned}
& (x p+2) q K_{2} \\
& (x p+3) q K_{3} \\
& =\dot{c}_{p-2} \\
& =c_{p-3} \\
& \because \\
& {[x p+(p-1)] q K_{p-1}=c_{1}} \\
& 2 q K_{2} \\
& \begin{aligned}
& =b_{p-2} \\
6 q K_{3} & =b_{p-3} \\
& \\
& \vdots \\
&
\end{aligned} \\
& (x+1) K_{1}+p(x+1) q \dot{K_{p}}=c_{0} \\
& -(p-1) p q^{2} K_{1}+(p-1) \cdot p q K_{p}=b_{0} \text {. } \\
& c_{p-1}=(x p+1) q K_{1} \\
& p(p+1) q K_{p+1}=\bar{b}_{1}-2\left(1+p q^{2}-p^{2} q^{2}\right) K_{2} \text {, }
\end{aligned}
$$

but in the case $p=2$ the last equation must be substitited by

$$
6 q K_{3}=2 q\left(1-2 q^{2}\right) K_{1}-2\left(1-2 q^{2}\right) K_{2} .
$$

Because the coefficients on the right-hand side of $(19 \mathrm{a})$ do not vanish, we can choose arbitrary complex values for the $(p-2)$ constants $K_{2}, K_{3}, \ldots, K_{p-1}$. The coefficients of $(19 \mathrm{~b})$ are real, and the determinant does not vanish. Therefore, we can give the constants $K_{1}$ and $K_{p}$ arbitrary real values. The general solution of (19) is

$$
\begin{aligned}
& c_{p-l}=(x p+l) q a_{l} \quad(l=1,2, \ldots, p-1), \\
& b_{p-k}=(k-1) k q a_{k}^{-} \quad(k=2,3, \ldots, p-1), \\
& c_{0}=(x+1)\left(a_{1}+p q a_{p}\right), \\
& b_{0}=(p-1) p q\left(-q a_{1}+a_{p}\right) ; . \\
& K_{i}=a_{i} \quad(i=1, \ldots, p), \\
& p(p+1) q K_{p+1}=\bar{b}_{1}-2\left(1+p q^{2}-p^{2} q^{2}\right) a_{2},
\end{aligned}
$$

where $a_{2}, \ldots, a_{p-1}$ are arbitrary complex constants and $a_{1}, a_{p}$ arbitrary real ones. For $p=2$ the last. equation must be substituted by

$$
6 q \dot{K}_{3}=2 q\left(1-2 q^{2}\right) a_{1}-2\left(1-2 q^{2}\right) \cdot a_{2} \text {. }
$$

Let $\varphi, \psi$ be holomorphic solutions of (14). Then the existence of such complex $a_{2}, \ldots, a_{j-1}$ and real $a_{1}, a_{p}$ is necessary for which the constan's $c_{i}, b_{j}$ and the first $(p+1)$ Taylor coefficients $K_{l}$ of $\varphi$ satisfy the relations $(20 \mathrm{a}),(20 \mathrm{~b})$. Henceforth, the constants $c_{i}, b_{i}$ are chosen in the sequel in accordance with (20a). 


\section{\$ 5 The complex differential equation for the function $\varphi$}

A. Taking into account $\psi^{\prime}(z)=\omega^{\prime}(z) \Psi^{\prime}(z)$, we have for $|z|<1$

$$
\bar{\omega}\left(\frac{1}{z}\right)\left(\frac{\varphi^{\prime}(z)}{\omega^{\prime}(z)}\right)^{\prime}+\psi^{\prime}(z)=\left(z^{2} \omega^{\prime}(z)\right)^{-1} R_{1}(z)
$$

and

$$
\frac{\varkappa}{z^{2}} \bar{\omega}^{\prime}\left(\frac{1}{z}\right) \frac{\varphi(z)}{\omega^{\prime}(z)}+\bar{\omega}\left(\frac{1}{z}\right) \frac{\varphi^{\prime}(z)}{\omega^{\prime}(z)}+\psi(z)=\left(z \omega^{\prime}(z)\right)^{-1} R_{2}(z) .
$$

Differentiating (21 b) and subtracting. (21a), one obtains the differential equation

with

$$
(x-1) Q(z) \varphi^{\prime}(z)+x Q^{\prime}(z) \varphi(z)=H(z) \text {. }
$$

$$
\dot{Q}(z)=\frac{1}{z^{2}} \frac{\bar{\omega}^{\prime}\left(\frac{1}{z}\right)}{\omega^{\prime}(z)}, \quad H(z)=\left(\frac{R_{2}(z)}{z \omega^{\prime}(z)}\right)^{\prime}-\frac{R_{1}(z)}{z^{2} \omega^{\prime}(z)}
$$

or

$$
\varphi^{\prime}(z)+\nu \frac{Q^{\prime}(z)}{Q(z)} \varphi(z)=\frac{1}{\alpha-1} Q(z)^{-1} H(z)
$$

with

$$
v=\frac{x}{x-1}
$$

The singularities of the linear differential equation (22) result from the poles and zeros of the functions $Q$ and $H$. Because of $0<|q|<1 / p$; the zeros of $Q(z)$ have simple multiplicity and are located at the $(p-1)$ complex roots $z_{1}, \ldots, z_{p-1}$ of $(-p q)$. The only pole of the functions $Q(z)$ and $H(z)$ is $z_{0}=0$. In this point we get the following Laurent's series:

$$
\begin{gathered}
Q(z)=\frac{1}{z^{p+1}} \cdot \frac{z^{p-1}+p q}{1+p q z^{p-1}}=\frac{p q}{z^{p+1}}+\left(1-p^{2} q^{2}\right) \frac{1}{z^{2}}+O(1) \text { for } p \geqq 3 \\
Q(z)=\frac{2 q}{z^{3}}+\left(1-4 q^{2}\right) \frac{1}{z^{2}}-2 q\left(1-4 q^{2}\right) \frac{1}{z}+O(1) \text { for } p=2
\end{gathered}
$$

and

$$
\begin{aligned}
& \frac{R_{1}(z)}{z^{2} \omega^{\prime}(z)}=\sum_{k=2}^{p-1} \frac{b_{p-k}}{z^{p-k+2}}+\frac{b_{0}}{z^{2}}+\left(\bar{b}_{1}-p q b_{p-2}\right) \frac{1}{z}+O(1) \\
& \left(\frac{R_{2}(z)}{z \omega^{\prime}(z)}\right)^{\prime}=-\sum_{k=1}^{p-1} \frac{(p+1-k) c_{p-k}}{z^{p-k+2}} \frac{c_{0}-\dot{p} q c_{p-1}}{z^{2}}+O(1)
\end{aligned}
$$

Bearing in mind the last two formulas and the relations (20a), one obtains by simple calculations the Laurent's expansion

$$
\begin{aligned}
H(z)= & -\sum_{l=1}^{p-1} p q[(p-l+1) x+l] \frac{a_{l}}{z^{p+2-l}}-\frac{1}{z^{2}} \\
& \times\left[(x+1)\left(1-p^{2} q^{2}\right) a_{1}+p q(x+p) a_{p}\right] \\
\quad & -\frac{1}{z}\left[(p-2)(p-1) q \bar{a}_{p-1}-2 p q^{2} a_{2}\right]+O(1)
\end{aligned}
$$


At first we consider the differential equation $\left(22^{\prime}\right)$ in the $z$-plane with non-intersecting branch cuts along the lines $\overparen{z_{0} \infty}, \widehat{z_{1} \infty}, \ldots, \widehat{z_{p-1} \infty}$. We define certain one-valued branches of the functions $Q(z)^{-\bullet}, Q(z)^{\gamma^{-1}}$ such that $Q(z)^{->} Q(z)^{-1}=Q(z)^{-1}$ holds. Thus, the coefficients in $\left(22^{\prime}\right)$ are. well-defined holomorphic functions within the unit circle with branch cuts. Consequently, in this domain we get the general solution of $\left(22^{\prime}\right)$ by

$$
\begin{aligned}
& \varphi(z)=C Q(z)^{-v}+\frac{Q(z)^{-v}}{x-1} \int Q(z)^{-1} H(z) d z, \\
& C-\text { arbitrary complex constant, }
\end{aligned}
$$

as a one-parameter family of analytic functions defined in the unit circle with branch cuts [1].

B. Obviously, the function $\varphi(z)$ of (25) cannot be holomorphic in the case $C \neq 0$, for the function $Q(z)^{-}$: has singularities at the points $z_{1}, \ldots, z_{p-1}$ because of $v>0$. Now we conșider the integral in (25). By virtue of (23), we have

$$
Q(z)^{->}=z^{(p+1),} \mathfrak{P}_{1}(z) \quad\left(\mathfrak{P}_{1}(0)=(p q)^{->} \neq 0\right)
$$

with a regular power series $\mathfrak{P}_{1}(z)$. Similarly, in consequence of $(23)$ and (24) one gets

$$
Q(z)^{v-i} \cdot H(z)=z^{-(p+1) \vee \mathfrak{q}_{2}(z)} \quad\left(\mathfrak{P}_{2}(0) \neq 0\right) .
$$

If we assume additionally that $(p+1) v$ is not an integer, then we can deduce

$$
\frac{Q(z)^{-}}{x-1} \int Q(z)^{\gamma-1} H(z) d z=z \Re_{3}(z) \quad\left(\mathfrak{P}_{3}(0) \neq 0\right)
$$

(This implies that the integral term of (25) is holomorphic in a sufficiently small neighbourhood of the origin. Analogously, we have in the neighbourhood of $z_{i}$ $(i=1, \ldots, p-1)$

$$
\begin{aligned}
& Q(z)^{-v}=\left(z-z_{i}\right)^{-\cdot} \mathfrak{P}_{4}\left(z-z_{i}\right) \quad\left(\mathfrak{P}_{4}(0) \neq 0\right) \\
& Q(z)^{-1} H(z)=\left(z-z_{i}\right)^{-1} \mathfrak{P}_{5}\left(z-z_{i}\right) . \quad\left(\mathfrak{P}_{5}(0) \neq 0\right)
\end{aligned}
$$

Therefore one gets .

$$
\frac{Q(z)^{-*}}{x-1} \int Q(z)^{r-1} H(z) d z=\mathfrak{F}_{6}\left(z-z_{i}\right)
$$

in a neighbourhood of the points $z_{i}(i=1, \ldots, p-1)$. For that reason, the solution (25) is holomorphic in the unit circle if and only if $C=0$, provided that $\nu(p+1)$ is not an integer. Introducing the notation

$$
\begin{aligned}
& r_{1}=a_{1}, \quad r_{2}=a_{p}, \quad r_{3}=\operatorname{Re} a_{2}, \quad r_{4}=\operatorname{Im} a_{2}, \ldots, r_{2 p-3}=\operatorname{Re} a_{p-2}, \\
& \dot{r}_{2 p-2}=\operatorname{Im} a_{p-2},
\end{aligned}
$$

the choice of parameters

$$
r_{j}=\delta_{i j}, \quad\left(j^{\prime}=1,2, \ldots, 2 p-.2\right)
$$

corresponds to $(2 p-2)$ linearly independent (in the real sense) holomorphic solutions $\varphi_{i}(z)(i=1,2, \ldots, 2 p-2)$ of $(25)$ : 


\section{$\$ 6$ Regularity of $\psi_{i}$}

In accordance with (21 b), we define the functions

$$
\begin{aligned}
& \psi_{i}(z)=\frac{1}{z \omega^{\prime}(z)}\left\{R_{2}^{(i)}(z)-\frac{x}{z} \bar{\omega}^{\prime}\left(\frac{1}{z}\right) \varphi_{i}(z)-z \bar{\omega}\left(\frac{1}{z}\right) \varphi_{i}{ }^{\prime}(z)\right\} \\
& (i=1,2, \ldots, 2 p-2),
\end{aligned}
$$

which are related to the holomorphic functions $\varphi_{i}(i=1, \ldots, 2 p-2)$. In this formula, we understand by $R_{2}^{(i)}(z)$ the function $R_{2}(z)$. with parameters (26). Since the $\varphi_{i}$ solve the differential equation (22), the $\psi_{i}$ are also solutions of (21 a)

$$
\bar{\omega}\left(\frac{1}{z}\right) \Phi_{i}{ }^{\prime}(z)+\psi_{i}{ }^{\prime}(z)=\frac{R_{1}^{(i)}(z)}{z^{2} \omega^{\prime}(z)} .
$$

We have still to check whether the functions $\psi_{i}$ are holomorphic in the unit circle. At first sight one realizes that the only singularity of $\psi_{i}$ can be placed in the origin. Furthermore, it is easily seen that $\psi_{i}$ is holomorphic within a neighbourhood of the origin if and only if the first $(p+1)$ coefficients $K_{1}^{(i)}, \ldots, K_{p+1}^{(i)}$ of the Taylor expan. sion of the functions $\varphi_{i}$ satisfy the relations

$$
\begin{aligned}
& K_{1}{ }^{(i)}=a_{1}, \ldots, K_{p}{ }^{(i)}=a_{p}, \\
& p(p+1) q K_{p+1}^{(i)}=\bar{b}_{1}-2\left(1+p q^{2}-p^{2} q^{2}\right) a_{2}
\end{aligned}
$$

with parameters (26). In order to check these conditions, we consider an arbitrary holomorphic solution $\varphi$ of (22). Let the Taylor series at the origin be

$$
\varphi(z)=K_{1} z+K_{2} z^{2}+\cdots+K_{p+1} z^{p+1}+O\left(z^{p+2}\right) .
$$

Using (23), one obtains by simple calculations

$$
\begin{aligned}
& (x-1) Q(z) p^{\prime}(z)+x Q^{\prime}(z) \varphi(z) \\
& =-\sum_{l=1}^{p-1} p q[x(p-l+1)+l] \frac{{ }^{\nu} K_{l}}{z^{p+2-l}} \\
& \quad-\frac{p q(x+p) K_{p}+(x+1)\left(1-p^{2} q^{2}\right) K_{1}}{z^{2}} \\
& \quad-\frac{(p+1) p q K_{p+1}+2\left(1-p^{2} q^{2}\right) K_{2}}{z} \text { for } p \geqq 3,
\end{aligned}
$$

but

$$
\begin{aligned}
& (x-1) Q(z) \varphi^{\prime}(z)+x Q^{\prime}(z) \varphi(z) \\
& =-2 q(2 x+1) \frac{K_{1}}{z^{3}}-\left\{2 q(x+2) K_{2}+(x+1)\left(1-4 q^{2}\right) K_{1}\right\} \frac{1}{z^{2}} \\
& \quad-\left\{6 q K_{3}+2\left(1-4 q^{2}\right) K_{2}-2 q\left(1-4 q^{2}\right) K_{1}\right\} \frac{1}{z} \text { for } p=2
\end{aligned}
$$

Consequently, the holomorphic solution $\varphi$ of (22) fulfills in every case the conditions (28). For proof the expansions (24) and (29a) (or (29b), respectively) must be compared, taking into consideration the parameter choice $(20 \mathrm{a}),(20 \mathrm{~b})\left(\left(20 \mathrm{~b}^{\prime}\right)\right)$. Hence we obtain with the parameters $(26)$ the $(2 p-2)$ linearly independent solutions $\varphi_{i}(z), \psi_{i}(z)(i=1,2,3, \ldots, 2 p-2)$ of the boundary value problem (14). 
Because the problem (14) is equivalent to the homogeneous boundary value problem (1), (2) (with $g=0$ ), we get the following interesting result:

Let $D$ be the domain to which the unit circle is mapped conformally by $\omega(z)=\cdot z$ $+q z^{p}(p \geqq 2,0<|q|<1 / p)$. Then the homogeneous problem (1), (2) permits exactly $(2 p-2)$ linearly independent solutions.

This result shows that the considered boundary value problem responds extremly sensible to a variation of the underlying domain. This is remarkable with respect to the simultaneous validity of Fredholm's theorems.

\section{$\$ 7$ Remarks}

The result of $\S 6$ holds true for every $0<|q|<\frac{1}{p}$. If $q$ converges to 0 , then the considered domain $D$ lies in an arbitrarily small vicinity of the unit circle. Thus, for every positive integer $n$ and in an arbitrary vicinity of the unit circle there exists such a domain for which the homogeneous problem (1), (2) permits at least $n$ linearly independent solutions. Finally, we consider the borderline case of the unit circle itself. Here the'considerations of $\$ \S 4-6$ can be repeated with $\omega(z)=z$. The complex boundary value problem is

$$
\begin{aligned}
& t \Phi^{\prime}(t)+t^{2} \Psi(t)-\frac{1}{t} \overline{\Phi^{\prime}(t)}-\frac{1}{t^{2}} \overline{\Psi(t)}=0 \\
& \frac{\varkappa}{t} \varphi(t)+\varphi^{\prime}(t)+t \psi(t)-\varkappa \overline{\varphi(t)}-\overline{\varphi^{\prime}(t)}-\frac{1}{t} \overline{\psi(t)}=0 \quad \text { for } \quad|t|=1 .
\end{aligned}
$$

Its solution depends on the number $2 v=\frac{2 x}{x-1}$. In every case we get the solution

$$
\varphi(\dot{z})=C z, \quad \psi(z)=0 . \quad(C-\text { real constant }) .
$$

These functions generate a radialsymmetric displacements field. If $2 v$ is an integer, then one obtains additionally 2 linearly independent solutions, which are the functions

$$
\varphi(z)=C_{1} z^{2 v}, \quad-\psi(z)=-\dot{C_{1}}(\varkappa+2 v) z^{20-2} .
$$

We have $2 v=2+\frac{1}{1-2 \sigma}$ in the case of plane deformation, but $2 v=1+\frac{2}{1-\sigma}$ for generalized plane stress. Provided that Poisson's ratio $\sigma$ is restricted by $\frac{1}{4} \leqq \sigma$ $<\frac{1}{2}$, the number $2 v$ can assume the integer values, $4,5,6, \ldots$ (for $\sigma=\frac{1}{4}, \sigma=\frac{1}{3}$, $\left.\sigma=\frac{3}{8}, \ldots\right)$ for plane deformation, but only the integer value $2 v=4$ for $\sigma=\frac{1}{3}$ in the case of generalized plane stress.

\section{REFERENCES}

[1] Bieberbach, L.: Theorie der gewöhnlichen Differentialgleichungen. Berlin -Göttingen Heidelberg - New York: Springer-Verlag 1965.

[2] Fichera, G.: Una introduzione alla teoria delle equazioni integrali singolari. Rend. di Matem. e delle sue Appl. (Ser. V) 17 (1958); 82-190. 
[3] Honsich, H.: Eine Verallgemeinerung der zweiten Randwertaufgabe. Monatsh. Math' Phys. 41 (1934), 445-450.

[4] JeNTsCH; L., and J. MaUL: Zur Elastizitäts- und Thermoelastizitätstheorie (Mathematica] Research: Bd. 4). Berlin: Akademie-Verlag 1980.

[5] КАлАндия, А. М.: Математические методы двумерноИ упругости. Москва: Физматгиз 1973.

[6] Mád, J.: Eine einheitliche Methode zur Lösung der ebenen Aufgaben der linearen' Elastostatik (Schriftenr. ZIMM Akad. Wiss. DDR) Berlin: Akademie-Verlag 1976.

[7] MaUL, J.: Uber ein bemerkenswertes Verhalten der Spannungen in der ebenen Elastizitätstheorie. ZAMM 57 (1977), 449-453.

[8] MaUL; J.: Uber das Poincarésche Problem der ebenen Elastizitätstheorie. Wiss. Z. KariMarx-Univ. Leipzig, Math.-Naturwiss. R. 25 (1976), 63-77.

[9] Muskrelishvil, I. N.: Some basic problems of the mathematical theory of elasticity (Transl. from the Russ.). Groningen: Noordhoff 1953.

[10] Moskhelishvir., I. N.: Singular integral equations (Transl. from the Russ.). Groningen: Noordhoff 1953.

[11] Schubert, H.: Uber das Randwertproblem von Poincaré in der Ebene. Wiss. Z. Univ. Rostock, Math.-Naturw. R. 19 (1970), $397-404$.

Manuskripteingang: 09. 04. 1984

\section{VERFASSER :}

Doz. Dr. sc. Johannes MádL

Sektion Mathematik der Karl-Marx.Universität

DDR -7010 Leipzig, Karl-Marx-Platz 